DOI: https://doi.org/10.24297/jns.v6i0.8449

\title{
Studying the Parameters and Reliability of The System of Small Intensity Irrigation for Work in The Conditions of Mining Agriculture in Azerbaijan.
}

\author{
Institute of Soil Science and Agrochemistry of the National Academy of Sciences of Azerbaijan \\ Prof. Dr. Z.H. Aliyev \\ zakirakademik@mail.ru
}

\begin{abstract}
Annotation:
The article discusses the issues of scientific and theoretical justification for the use of automated irrigation in agriculture, that these systems can reduce the capital costs of their construction, as well as ensure the principle of "rain" and "drip irrigation" with daily water consumption of plants. There are optimal conditions for the growth and development of plants during their growing season. It is proved that the automated irrigation of agricultural crops establishes high yields and increased labor productivity. Therefore, the elements and devices of the used automatic machine require reliable and uninterrupted operation. This is due to the fact that the problem is that the problem lies not only in technical reliability but also in economic efficiency., including micro-irrigation systems. Reliability, performance, and performance. Initially, the existing irrigation technique, stationary irrigation system consisted of a large number of elements of the same type, necessary for conducting field cultural events.
\end{abstract}

Keywords: System Tasks, Automated Irrigation, Operational Indicators. Reliability, Irrigation Rate, Drip Irrigation, Sprinkling, Etc.

\section{INTRODUCTION}

In recent years, both in the republic and in the CIS and abroad, the development of stationary systems of lowintensity irrigation, consisting of micro-sprinkling, pulse sprinkling of self-oscillating action, aerosol irrigation, combined sprinkling, drip, pulsed-drop and a number of others. This is due to the fact that the low-intensity irrigation system has a number of significant advantages over other irrigation methods. It is especially promising to create automated sprinkler systems using impulse devices, combined micro-sprinklers, rocker-type step micro-sprinklers, self-oscillating pulse-droplet devices, etc. It should be noted that these systems can reduce capital costs for their construction, as well as ensure the principle of both sprinkling" and "drip irrigation" with daily water consumption of plants. That is, to create optimal conditions for the growth and development of plants during their growing season. Automated irrigation of crops is the basis of high yields and increased labor productivity. Therefore, the elements and devices of the applied automation require a reliable and uninterrupted operation. Therefore, starting from the development stage of these devices and ending with production and operation, there are reliability issues, since the reliability problem is not only a technical but also an important economic task.

\section{Objectives of research and discussion of materials:}

Reliability theory developed in recent decades offers great opportunities for a qualitative assessment of the reliability of existing irrigation equipment, including micro-irrigation systems. Reliability is the property of an equipment or system to perform specified functions, to maintain its performance over the required period of time or required operating time.

Among the existing irrigation equipment, a stationary sprinkler system consisting of a large number of similar elements located in a certain order on the field and necessary for irrigation of crops. Determining the reliability of one of these elements solves the issue of system reliability. The reliability issues of stationary irrigation systems, in particular, those equipped with sprinklers, were dealt with by many scientists in the former Soviet Union and abroad. To study the elements of calculation on the reliability of low-intensity irrigation systems 
(using pulsed irrigation of self-oscillating action as an example), the technological and feasibility study of irrigation systems should be considered.

The optimal parameters of the low-intensity irrigation system were determined by us by searching for the minimum of the function of specific reduced costs Court. depending on the area of the system S; aspect ratio $\theta$; $\mathrm{Q}$ system costs; head $\mathrm{H}$, corresponding to the pressure of the dictating point; conditional irrigation period $\mathrm{Tu}$, equal to where, $M$ irrigation rate, $\mathrm{m}$; $\mathrm{q} /$-maximum ordinate of the hydraulic module $\mathrm{m} / \mathrm{s}$ ).

Numbers of distribution pipelines $\mathrm{N}$; distance between irrigation equipment (sprinkler plants) I; coefficient $\mathrm{X}$, equal when placing devices on a square of 0.2 ; the length of the $i$-th pipeline of the system li of its diameter $\mathrm{Di}$ and flow rate $\mathrm{Qi}$, coefficients $k, m, \beta$ depending on the roughness of the inner surface of the pipes; cost of

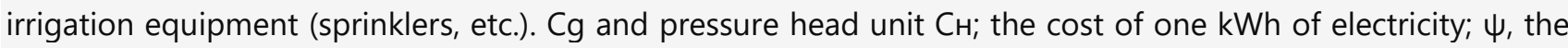
efficiency of the pump unit $\eta$, equal to 0.7 coefficient of an uneven flow

$$
K_{H}=\frac{2 r l_{n} p}{(p-1)(r+1)}
$$

where, $r$ is the ratio of the pressure of the beginning of the surge $P 2$ to the pressure of the end of the surge $P 1$ ), equal to $1.02-1.25$; coefficient E0 = E1 + E2 (E1, E2 - standard coefficients of efficiency of capital investments and annual deductions for depreciation and repair); the coefficients $b$, d depending on the material and the cost of laying pipes and operating costs for the maintenance of personnel of the En system:

$$
C n д=-\left[E O \sum(a+b D i a) l+\left(C+\sum C g\right)+B Q\left(H+\sum K Q i \beta D i-m l i\right)+E n\right]
$$

Studies have shown that the most economical option is the simultaneous operation of all sprinklers of the system, i.e., when the limiting dispersion of irrigation current is performed on the system. Function (2) for this case has a minimum at the points defined by the expressions:

$$
\begin{gathered}
N=\frac{n 2^{H n}}{1-n} ; \quad S=\frac{Q^{\frac{1+n}{2}} S^{\frac{1-n}{2}}}{l^{1-n}} \\
L=\left[\frac{\gamma \lambda(1-n)^{1+n}}{x B\left(n^{2 n}\right)^{n}}\left(q^{\prime}\right)^{n-1}\left(\frac{S}{\theta}\right) \frac{n^{2}}{2}\right]^{(n 2-2 n+2)^{-1}} \\
\text { Where, } n=\frac{\alpha(\beta+1)}{\alpha+m} \quad \lambda=(m+\alpha)\left(\frac{B \kappa}{\alpha}\right)^{\frac{\alpha}{\alpha+m}}\left(\frac{E_{o} b}{m}\right)^{\frac{m}{\alpha+m}} \\
\gamma=1-\frac{n}{2}-\frac{n(1-n)}{6} \quad B=\frac{1000}{102 \eta} \psi K H
\end{gathered}
$$

Sub

adding coefficients corresponding to steel pipelines in formulas (.3) and (4) $(d=1.4 ; m=5.1 ; \beta=1.8 ; K=$ $0.00107 ; \mathrm{B}=50 ; \mathrm{EO}=0.2 ; \mathrm{Kn}=1,1$ ), we obtain the calculation formulas for the determination and which, for the convenience of their use, are represented by nomograms (Fig. 1).

Anologous nomograms are also constructed for pipes made of plastics and other materials. 
The optimal diameters of pipelines Dopt, I, the number of irrigation pipelines $X$, suspended from the distribution pipe, the number of sprinklers on the irrigation pipe $y$ is determined by the following ratios:

$$
D o \pi \tau=\left(\frac{B K m}{E o \alpha b}\right)^{\frac{1}{\alpha+m}} Q^{\frac{\beta+1}{\alpha+m}} \quad \chi=\frac{1}{2 l} \sqrt{\frac{S}{\theta}} \quad y=\frac{2 \sqrt{S \theta}}{N l}
$$

When changing the initial parameters $S, q /, \theta, M$ ) within:

$$
\begin{aligned}
& 30<=\mathrm{S}<=300 \text { ha, } 0.310-7<=\mathrm{q} /<=1.0 \times 10-7 \mathrm{~m} / \mathrm{s}, \\
& 0.25<=\theta<=4,0.3<=\mathrm{M}<=1.0 \mathrm{~m}
\end{aligned}
$$

$1000 \mathrm{~m} 3$ / ha, the optimal system parameters vary within:

$35<=\mathrm{I}<=135 \mathrm{~m} ; 3<=\mathrm{N}<=65 ; 1<=\mathrm{x}<=50 ;$ and $1<=\mathrm{y}<=33$

Attaching to the areas assigned to specific natural and economic conditions, we determine the parameters of low-intensity irrigation systems using the example of pulsed sprinkling.

We consider these areas to be established and equal to 150 - 400 hectares and are characterized on average by an optimal aspect ratio of 1-3, and environmental conditions with an irrigation norm of $0.35-0.75 \mathrm{~m}$ and a maximum ordinate of the hydraulic module of $0.6 \times 10-7-1,0 \times 10-7 \mathrm{~m} / \mathrm{s}$. Averaged initial data $\mathrm{S}=200 \mathrm{ha}, \theta$ $=2 ; \mathrm{M}=0.55 \mathrm{~m}, \mathrm{q} /=0.8 \times 10-7 \mathrm{~m} / \mathrm{s}$, the following optimal system parameters $\mathrm{I}=50 \mathrm{~m}, \mathrm{~N}=22, \mathrm{x}=10, \mathrm{y}=8$ obtained using the previously given program and formulas (4.5)

For other cultivated crops and environmental conditions, these parameters are determined similarly.

The obtained values of $N, I, x, y, D$ opt. I - allow you to determine the main structural and technological parameters of irrigation equipment (pulse sprinklers of self-oscillating devices): as a pressure $\mathrm{H} 2$, corresponding to the start pressure of the surge $\mathrm{P} 2$, pressure $\mathrm{H} 1$, corresponding to the pressure of the end of the surge $\mathrm{P} 1$; Pneumatic accumulator design volume $\mathrm{W} 0$ and nozzle diameter $\mathrm{D}$.

Based on the materials of a number of works by scientists of the World, and the results of studies conducted at the Institute of Erosion and Irrigation of ANAS devoted to the determination of some parameters of irrigation equipment, including conventional sprinklers, pulsed devices, etc., according to which we propose the following characteristic controls for determining the main parameters of pulse devices depending on the pressure. But, corresponding to the initial pressure in the pneumatic accumulator P0 and the corresponding atmospheric pressure Ra, the cross-sectional areas of the supply pipe $\omega 2$, the inlet flow coefficients $\mu 1$ and the nozzle $\mu 2$, the resources of the pneumatic accumulator $T *$, the sprinkler apparatus $N *$, and coefficients that depend on the cost of the sprinkler apparatus $\mathrm{d} 1$ and $\mathrm{d} 2$,

$$
\begin{aligned}
& \frac{H_{o} W_{o}\left(H_{2}-H_{1}\right)}{H_{1} H_{2}\left(T_{1}+T_{2}\right)}=k_{1} q^{\prime} l^{2} \\
& \mathrm{~W} 0=\mathrm{R}^{2}=\sqrt{\left.\frac{\boldsymbol{a}_{1} \boldsymbol{p} \boldsymbol{M} \boldsymbol{H}_{1} \boldsymbol{H}_{2} \boldsymbol{T} *}{\boldsymbol{H}(\boldsymbol{H}}-\boldsymbol{H}_{1}\right) \boldsymbol{a}_{2} \boldsymbol{N} *}
\end{aligned}
$$




$$
\mathrm{D}=\left[\frac{H_{1}^{0.43}}{6.6} \cdot \frac{H_{2} / H_{1}-1}{\left(H_{2} / H_{1}\right)^{0.57}-1}\right]^{7.15} \cdot 10^{-2}
$$

It should be noted that equation (6) correlates the water supply and water consumption of plants, and equation (7) and (8) characterize, respectively, the optimal sizes of sprinklers and agrotechnical requirements and the quality of the rain (the number of sprinklers with a diameter of more than $1.0-1,5 \mathrm{~mm}$ in the total flow of the sprinkler stream should be less than $10 \%$ ).

Each group $M, q, S, \theta$ corresponds to well-defined values of the $x, y, N, I$ parameters of the system and $H 2 H 1$, W0, D parameters of the pulse apparatus. Taking into account that on a real system $T 1=>T 2$ and the sum of hydraulic resistances to the worst according to the filling conditions of the pneumatic accumulator of the system $\sum \xi=>1$, equation (6) can be represented as:

$$
\begin{aligned}
& H I=\sqrt{\left(\frac{H_{2}^{2}+2 C^{2} H_{2}}{2 C^{2}}\right)^{2}+\frac{H_{2}^{3}-C^{2} H^{2}}{C^{2}}}-\frac{H_{2}^{2}+2 C^{2} H_{2}}{2 C^{2}} \\
& C=\frac{k_{1} q}{\mu_{1} \omega_{1} \sqrt{2 g}} \quad \mu=\sqrt{\frac{\left(A q^{n_{1}}\right)^{1.3}}{\gamma a_{1} l F(x \cdot y \cdot N)}} \quad A=\left(\frac{B k m}{E_{o} \alpha b}\right)^{\frac{1}{\alpha+m}} \\
& F(x . y \cdot N)=y^{1-n_{2}}+x \frac{1-n_{2}}{1.3 n_{1}}+\frac{N^{1-n_{2}} y^{1-1.3 n}}{x^{1.3 n_{1}} 2^{1.3 n_{1}-n_{2}+1}} q=q^{1} l^{2} \\
& \omega=0.785\left(A q^{n_{1}}\right)^{2} n_{1}=\frac{\beta+1}{\alpha+m}=0.44 / n_{2} /=/ 2-5,3 n_{1} /=0,33
\end{aligned}
$$

Qi coefficient equal to 2.64 for steel pipes. $10-3 /\left(2,64.10^{-3} /\right)$

Equations (7.8 and 9) together with the formula A.P. Rusetskii represent a closed system of equations.

Figure 2 below shows a graphical solution to this system.

Knowing the economic radius of action of the pulse apparatus and the parameter $C$ of the system $(C=3-5)$, the solution should be searched for according to the corresponding $C$ curves in the following order by $a 1$ a4, a7, a10 by $C=3$, by a2, a5, a8, a11 at $C=4$ and along a3, a6, a9, a12 at $C=5$.

The parameters of the sprinkler apparatus at intermediate values of $C$ are determined by interpolation. 

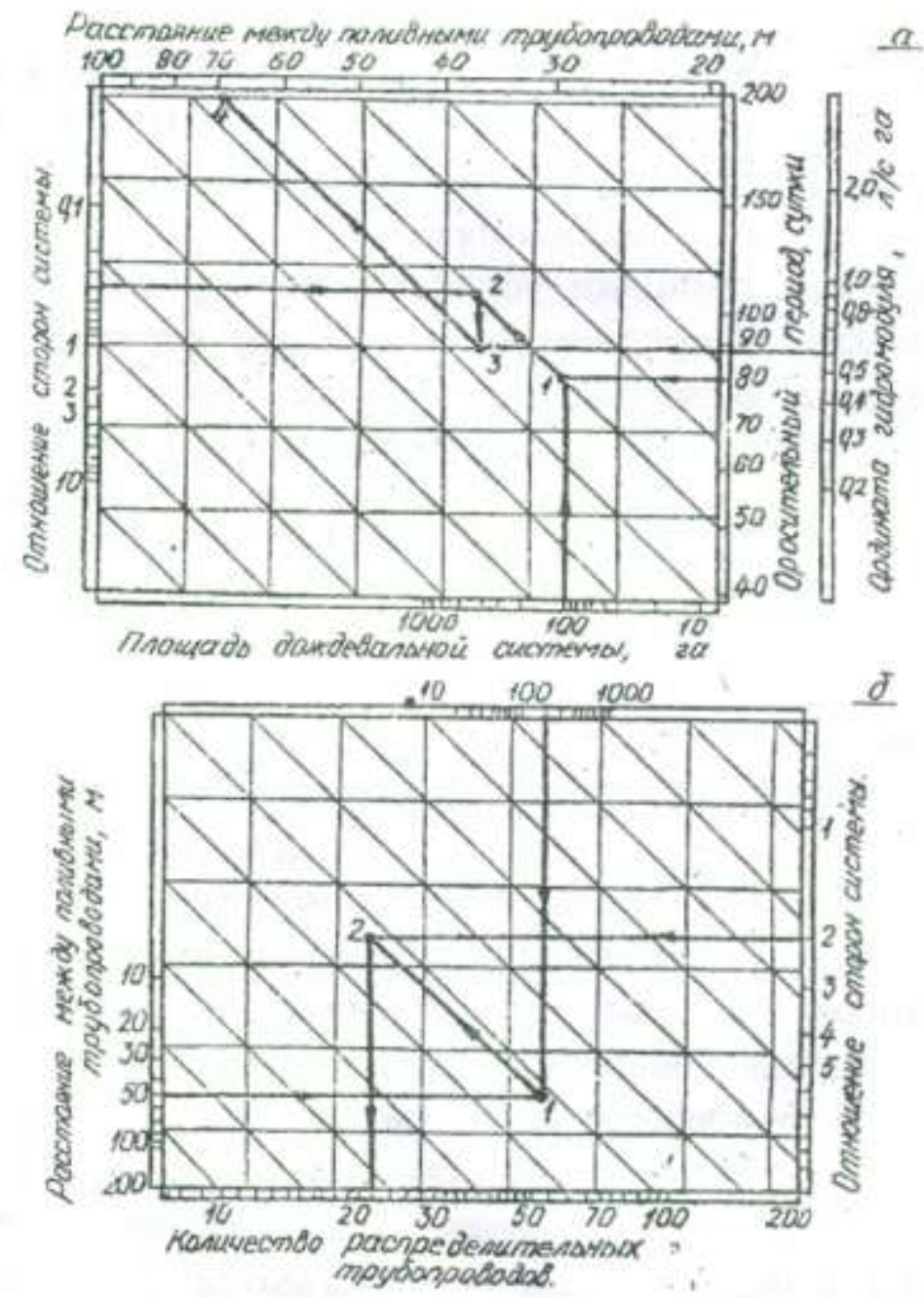

Fig. 2 Nomogram for determining the parameters of a pulse apparatus.

Using the results obtained earlier, we determine the design and technological parameters of the pulse apparatus for the same environmental conditions. When arranging devices by squares, its economical radius of action is $R$ $=21-35$, and the parameter $C=3$. Using the nomogram (Fig. 2) and the input quantities $\mathrm{K}$ and $\mathrm{C}$ The following parameters of the pulse apparatus are obtained:

$$
P_{1}=450 \mathrm{kPa}, \quad P_{2}=700 \mathrm{kPa}, \quad W_{0}=0.19 \mathrm{~m}^{3}, \quad D=20 \mathrm{~mm} .
$$

To solve a set of measures for the reliability of systems is the reliability of the elements of sprinkler systems.

Investigations of the reliability indicators of the main elements of the stationary sprinkler system are given the results of theoretical and experimental studies, the noise immunity of the telecontrol devices is estimated, the dispersion parameters of the irrigation norms precipitated by individual sprinklers are determined, and the types and parameters of the distribution of operating times to the failure of the main elements of the sprinkler systems are determined.

At the moments when the telecontrol device accepts the command "object selection," transient processes occur in the network of technological pipelines. 
It was found that the pressure $P$, in this case, can take values of a smaller value of the statistical pressure of the reconfiguration of the telecontrol device $\mathrm{P} / 0$ and the duration of the false signal can reach $0.15-1.2$ seconds.

The following experimentally confirmed dependence was obtained between the processing time of the command signal $t k$, the stroke of the rod $h$, the effective area of the membrane Fe, the cross-sectional area of the tube connecting the process pipe to the hydraulic drive cavity $\omega$, the stiffness coefficient of the return spring $\mathrm{K} 2$, the specific gravity of the water $\gamma \mathrm{b}$ and the acceleration of gravity $\mathrm{g}$ :

$$
t_{k}=\frac{F_{\ni}}{\mu \omega k_{2}} \sqrt{\frac{2 F_{\ni} \gamma b}{g}}\left[\sqrt{h K_{2}+F_{\ni}\left(P_{o}^{\prime}-P\right)} \sqrt{F_{\vartheta}\left(P_{o}^{\prime}-P\right)}\right]
$$

According to the formula (10), the time for reconfiguring the automated telecontrol device was estimated.

With an instant drop. the pressure in the cavity of the hydraulic actuator to atmospheric from a pressure of 340 $\mathrm{kPa}$, corresponding to the pressure "object selection," it takes $0.34 \mathrm{~s}$, which is 1.5 times the duration of the false signal.

Dependencies (10) allows not only to estimate the time of reconfiguration of the device for automated control of the technological process of irrigation through the channel of telecontrol systems but also to assign its design value to the parameters in accordance with the requirements of noise immunity of elements of telecontrol systems

The dispersion of the pressure of the beginning of the outburst of pulsed self-oscillating devices leads to the dispersion of their outburst volumes $\Delta \mathrm{W}$, therefore, of the rain layer $\mathrm{M}$, since they are related by $\Delta W=\frac{P_{o} W_{o}\left(P_{2}-P_{1}\right)}{P_{1} P_{2}}$ and $M=\Delta W \frac{N_{o}^{\#}}{S_{1}}\left(N_{o}^{\#} \cdot S_{1}\right)$ (the number of machine operation cycles per year and its service area).

Tests of pulsed devices (sample 100) and subsequent processing of the data obtained showed that the distributions of $P_{2}$ and $P_{1}$ obey the normal law with variation coefficients $v p_{2}=0.04$ and $v p_{1}=0.093$. I mean,

$$
\text { as well } \quad \bar{M}=\frac{\Delta W N_{o}^{\#}}{S_{1}} \quad \delta_{m}=\frac{N_{o}^{\#}}{S_{1}} \delta_{\Delta w}
$$

the distribution density $\mathrm{M}$ can be represented as:

$$
f(M)=\frac{\Delta \bar{W}}{\sqrt{2 \pi} M \delta_{\Delta w}} \exp \left[-\frac{(\Delta W-\Delta \bar{W})^{2}}{2 \delta_{\Delta w}^{2}}\right]
$$

Distribution (11) can be used in assessing damage from the structural imperfection of pulse devices.

Obviously, the smaller the ratio, the more perfect the apparatus. $\frac{\delta_{\Delta w}}{\Delta W}$

Studies (Table 1) were carried out to determine the types and parameters of the distribution of mean time to failure (, $\sigma$ is the mean and standard deviation) of typical irrigation system devices that include the most common elements in hydraulic automation: underground retractable hydrants (elements 1.,2), devices of automated remote control systems (elements 3., 4), rotary mechanisms (and element 5) as well as pulsed sprinklers (elements 6,7, 8, 9). 
It is established that the distribution of the recovery time of these elements can be characterized by the average recovery time of TV.

As a result of special studies, the following relationship was obtained between the average service life of parts made of rubber or plastic Tcr, the average number of cycles $\mathrm{N} \# 0$ per year and the experimental value of the aging parameter of these materials in water T0, equal to 6.8 years:

$$
T_{c p}=\frac{N_{o} T_{o}}{N_{o}+N_{o}^{\#} T_{o}}
$$

Table 1

\begin{tabular}{|c|c|c|c|c|c|}
\hline \multicolumn{6}{|c|}{ Medium-water, $\mathrm{t}^{0}$ of medium $15-20^{\circ} \mathrm{C}$, cyclic load 200-800 kPa } \\
\hline \multirow{2}{*}{ Items } & \multirow{2}{*}{ Material } & \multirow{2}{*}{$\begin{array}{l}\text { Characteristic } \\
\text { of double stroke } \\
\text { lengths, } \mathbf{m m}\end{array}$} & \multirow{2}{*}{$\begin{array}{l}\text { Distribution } \\
\text { law }\end{array}$} & \multicolumn{2}{|c|}{ Distribution Parameters } \\
\hline & & & & $\begin{array}{l}\mathrm{N}_{(\mathrm{\tau})} \text { cycle } \\
10^{-3}\end{array}$ & $N_{E, r}$ \\
\hline $\begin{array}{l}\text { 1. Collar } 200 \text { GOST } \\
5678-72\end{array}$ & $\begin{array}{l}\text { Rubber } 4004 \\
\text { MRTU 38-5-1166- } \\
\text { 54 }\end{array}$ & Abrasive wear, 140 & Normal & $0,76(0,14)$ & 3,56 \\
\hline $\begin{array}{l}\text { 2. Cuff } 280 \times 320 \\
\text { GOST } 6969-74\end{array}$ & 《 --. --. & «--------» & « -» & $1,25(0.23)$ & 2,45 \\
\hline $\begin{array}{l}\text { 3. Membrane } 50 \\
\text { GOST } 9887-78\end{array}$ & $\begin{array}{l}\text { Rubber } 3825 \\
\text { MRTU 38-5-1166- } \\
54\end{array}$ & Fatigue wear: 30 & « -» & $6,7(1,45)$ & 1,25 \\
\hline $\begin{array}{l}4 . \quad \text { Ratchet } \\
\text { mechanism }\end{array}$ & « --- --- » & Jamming & Exponential & $4,2(4,2)$ & 0,25 \\
\hline $\begin{array}{l}\text { 5. Membrane } 50 \\
\text { JOST 9887-78 }\end{array}$ & $\begin{array}{l}\text { Rubber } 3825 \\
\text { MRTU 38-5-1166- } \\
\text { 54 }\end{array}$ & Fatigue wear: 10 & Normal & 1500(500) & 0,25 \\
\hline
\end{tabular}

In addition to these measures, organizational measures should be developed to address the optimal level of operational reliability of stationary systems. For organizational activities, the implementation of preventive work and the correct deadlines.

Determining the timing of preventive maintenance is one of the main problems of preventive maintenance, closely related to the content of preventive work and the organization of their implementation. These terms are usually determined by examining the following objective function: 


$$
C(t)=\frac{C_{1} M(n)+C_{2} N(t)}{t}
$$

where, $C 1, C 2$, respectively, the cost of replacing parts when eliminating failure and preventive replacements;

$M(t), N(t)$ - respectively, the average number of replacements in case of failure and preventive maintenance during time t.

The indicator of the assessment of perfection derived by us is advisable to apply with the aim of choosing the most advanced design since it economically summarizes the indicators of maintainability, persistence, and safety of sprinkler systems.

\section{Literary sources:}

1. Aliev B.H., Aliev Z.H and others Techniques and technology few intensive irrigations in condition of the mountain region Azerbaijan. Publishers "Elm," Baku, 1999, p. 220.

2. Aliev B.H., Aliev Z.H. The premises about the most important problem of the agriculture in provision water resource mountain and foothill regions Azerbaijan, J. AAS, \# 1-3, Baku, 2007, p. 179-182.

3. Aliev B.H., Aliev Z.H. The premises of the decision of the problems moisture provides agriculture cultures production in mountain and foothill region Azerbaijan. The works SRI "Erosions and Irrigations." Baku, 1999, p. 125-129.

4. Aliev B.G., Aliev Z.G. Zoning of the territory of the Republic of Azerbaijan for the selection of advanced irrigation technologies. Monograph, Zia-Nurlan Publishing House, Baku, 2001, p. 249.

5. Huseynov G.M. Ways to increase the efficiency of irrigated land use, improve technology and methods of irrigation of agricultural crops in Azerbaijan. Competition degree report. C. s. D. based on work, Baku, 1969, p.30.

6. International Center for Agricultural Research in arid regions in arid regions (ICARDA) Irrigation regime and monitoring technique. Edited by U. Umarov and A. Karimov. Taraz: IC "AQUA", 2002, $128 \mathrm{~s}$.

7. V.F. Nosenko Irrigation in the mountains. Publishing House Kolos Moscow 1981.143 p. 